\begin{tabular}{|c|c|c|c|c|c|}
\hline \multirow{3}{*}{$\begin{array}{l}\text { 2. ECN Category (mark one) } \\
\text { Supplemental } \\
\text { Direct Revision } \\
\text { Change ECN } \\
\text { Temporary } \\
\text { Standby } \\
\text { Supersedure } \\
\text { CancelNoid }\end{array}$} & \multicolumn{3}{|c|}{$\begin{array}{l}\text { 3. Originator's Name, Organization, MSIN, and Telephone No. } \\
\text { D.A. Barnes, Data Development and } \\
\text { Interpretation, R2-12, } 373-3667\end{array}$} & $\begin{array}{l}\text { 4. USQ Required? } \\
\text { Yes O No }\end{array}$ & $\begin{array}{l}\text { 5. Date } \\
2 / 28 / 2000\end{array}$ \\
\hline & \multicolumn{2}{|c|}{$\begin{array}{l}\text { 6. Project Title/No. Work Order No. } \\
\text { Tank Monitor and Control System }\end{array}$} & \multicolumn{2}{|c|}{$\begin{array}{l}\text { 7. Bldg./Sys./Fac. No. } \\
2750 \mathrm{E}, \mathrm{B} 105\end{array}$} & \\
\hline & \multicolumn{2}{|c|}{$\begin{array}{l}\text { 9. Document Numbers Changed by this ECN (includes } \\
\text { sheet no. and rev.) }\end{array}$} & \multicolumn{2}{|c|}{$\begin{array}{l}\text { 10. Related ECN No(s). } \\
\text { N/A }\end{array}$} & 11. Related PO No. \\
\hline $\begin{array}{l}\text { 12a. Modification Work } \\
\text { Yes (fill out Blk. 12b) } \\
\text { No (NA Blks. 12b. } \\
\qquad \begin{array}{l}\text { 2c, 12d) } \\
\text { (n) }\end{array}\end{array}$ & $\begin{array}{l}\text { 12b. Work Package No. } \\
\text { N/A }\end{array}$ & \multicolumn{2}{|c|}{$\begin{array}{l}\text { 12c. Modification Work Completed } \\
\text { N/A } \\
\text { Design Authority/Cog. Engineer Signature \& }\end{array}$} & \multicolumn{2}{|c|}{$\begin{array}{l}\text { 12d. Restored to Original Condition (Temp. } \\
\text { or Standby ECNs only) } \\
\text { N/A } \\
\text { Design Authority/Cog. Engineer Signature \& }\end{array}$} \\
\hline
\end{tabular}

13a. Description of Change

13b. Design Baseline Document? $\bigcirc$ Yes $\bigcirc$ No

Complete revision of document. (Incorporates ECN 655999)

14a. Justification (mark one)
Criteria Change
Design Impravement
Environmental
Facility Deactivation
As-Found
Facilitate Const.
Const. Error/Omission
Design ErrorrOmission

15. Distribution (include name, MSIN, and no. of copies)

See attached distribution. 


\section{ENGINEERING CHANGE NOTICE}

16. Design Verification Required

\section{O Yes \\ ONo}

17. Cost Impact

ENGINEERING

$\begin{array}{ll}\text { Additional } & \$ \\ \text { Savings } & \$\end{array}$

\begin{tabular}{ll} 
& \multicolumn{2}{c}{ CON } \\
Additional & $\mathrm{s}$ \\
Savings & $\mathrm{S}$
\end{tabular}

Page 2 of 2

18. Schedule Impact (days)

Improvement

Delay

19. Change Impact Review: Indicate the related documents (other than the engineering documents identified on Side 1) that will be affected by the change described in Block 13. Enter the affected document number in Block 20.

$\begin{array}{llll}\text { SDD/DD } & \square & \text { Seismic/Stress Analysis } \\ \text { Functional Design Criteria } & \square & \text { Stress/Design Report } & \square \\ \text { Operating Specification } & \square & \text { Interface Control Orawing } \\ \text { Criticality Specification } & \square & \text { Calibration Procedure } & \square \\ \text { Conceptual Design Report } & \square & \text { Installation Procedure } & \square \\ \text { Equipment Spec. } & \square & \text { Maintenance Procedure } & \square \\ \text { Const. Spec. } & \square & \text { Engineering Procedure } & \square \\ \text { Procurement Spec. } & \square & \text { Operating instruction } & \square \\ \text { Vendor Information } & \square & \text { Operating Procedure } & \square \\ \text { OM Manual } & \square & \text { Operational Safety Requirement } \\ \text { FSAR/SAR } & \square & \text { IEFD Drawing } & \square \\ \text { Safety Equipment List } & \square & \text { Cell Arrangement Drawing } & \square \\ \text { Radiation Work Permit } & \square & \text { Essential Material Specification } \\ \text { Environmental Impact Statement } & \square & & \square \\ \text { Environmental Report } & \square & \text { Fac. Proc. Samp. Schedule } & \square \\ \text { Environmental Permit } & \square & \text { Inspection Plan } & \square\end{array}$

\begin{tabular}{lll}
$\square$ & Tank Calibration Manual & $\square$ \\
$\square$ & Health Physics Procedure & $\square$ \\
$\square$ & Spares Multiple Unit Listing & $\square$ \\
$\square$ & Test Procedures/Specification & $\square$ \\
$\square$ & Component Index & $\square$ \\
$\square$ & ASME Coded Item & $\square$ \\
$\square$ & Human Factor Consideration & $\square$ \\
$\square$ & Computer Software & $\square$ \\
$\square$ & Electric Circuit Schedule & $\square$ \\
$\square$ & ICRS Procedure & $\square$ \\
$\square$ & Process Control Manual/Plan & $\square$ \\
$\square$ & Process Flow Chart & $\square$ \\
$\square$ & Purchase Requisition & $\square$ \\
\hline & Tickler File & $\square$
\end{tabular}

20. Other Affected Documents: (NOTE: Documents listed below will not be revised by this ECN.) Signatures below indicate that the signing organization has been notified of other affected documents listed below.

\section{Document Number/Revision}

Document Number/Revision

Document Number/Revision

$\mathrm{N} / \mathrm{A}$

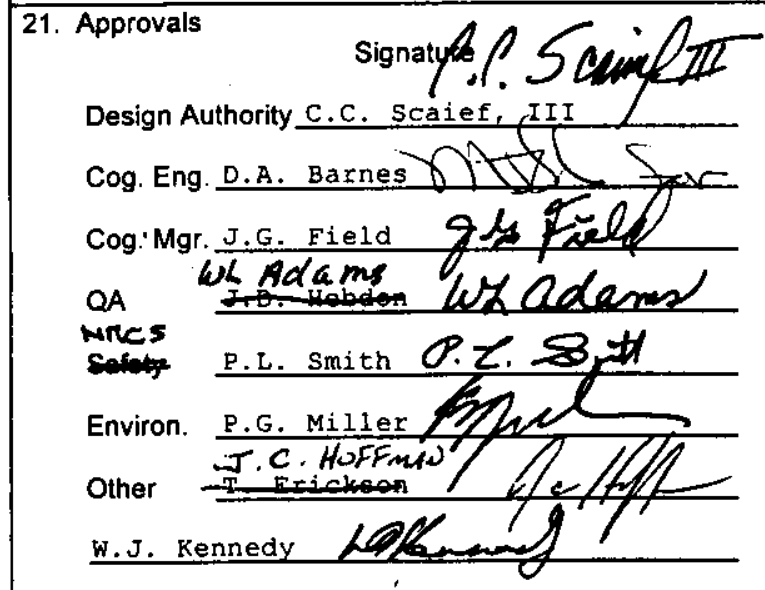

Date $3-1-00$ $3-1-8 \%$ $3-1-00$ $3-2-00$ $3 / 2 / 00$ $\frac{3 / 2 / 00}{3-2-00} \frac{3 / 2 / m}{3 / 0}$ DEPARTMENT OF ENERGY

Signature or a Control Number that tracks the Approval Signature

Design Agent

PE

QA

Safety

Design

Environ.

Other

\section{ADDITIONAL}

Signature

Date

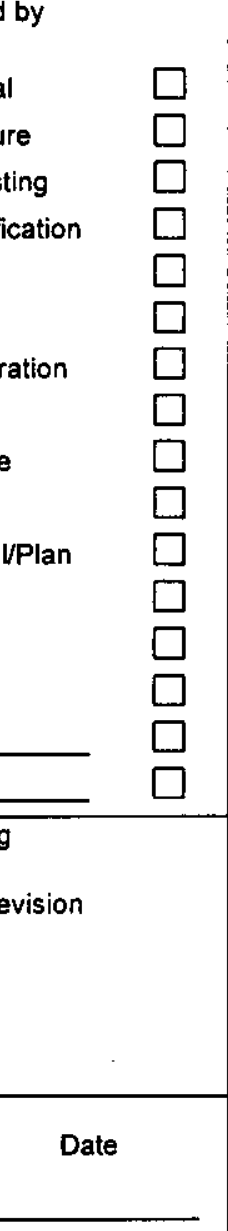




\section{DISTRIBUTION SHEET}

To

Distribution

Project Title/Work Order

Tank Monitor and Control System (TMACS) Version Description Document (VDD)

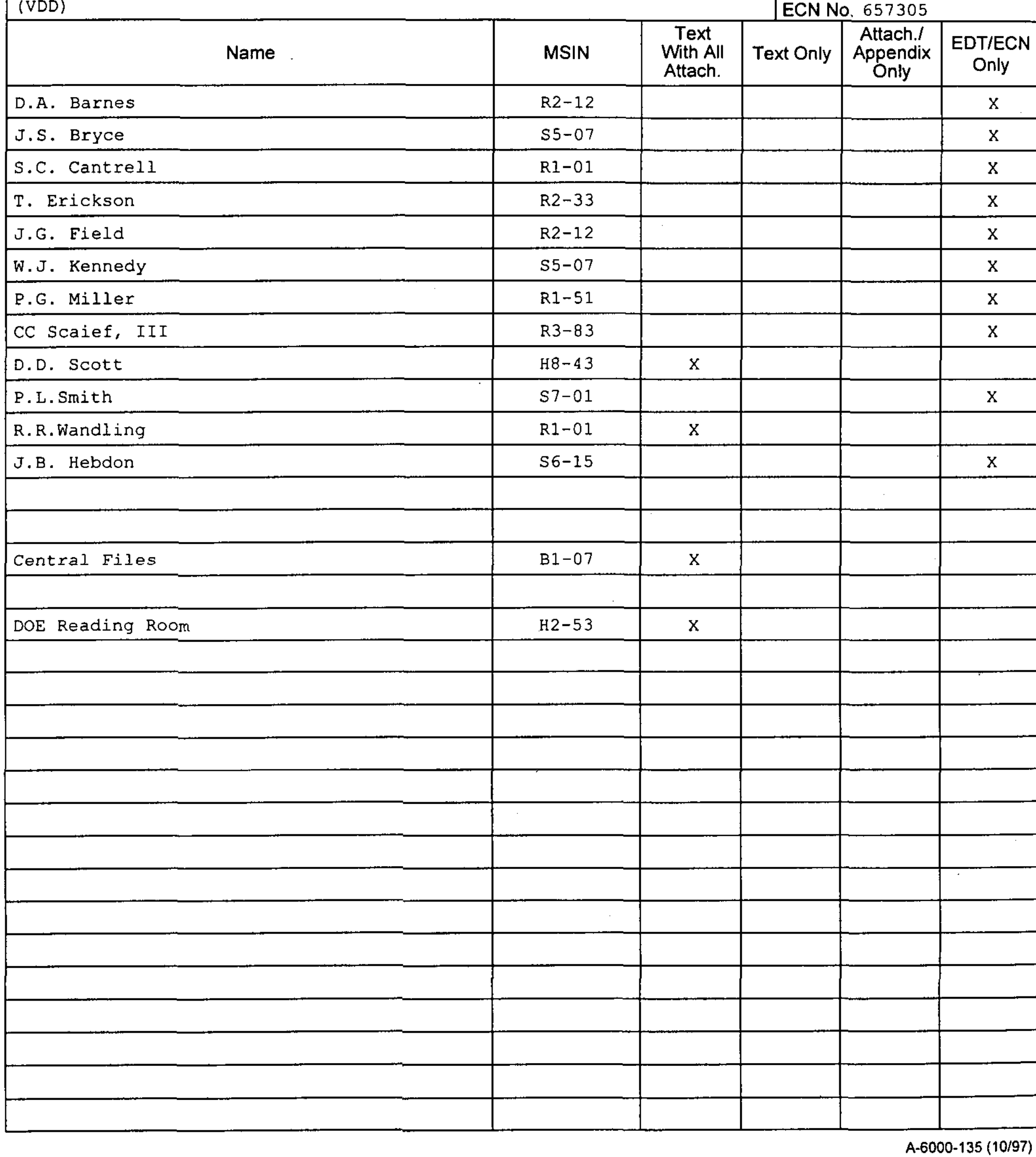

Page 1 of 1 Date 2/28/2000

EDT No. N/A

ECNNO 657305

From
Data Development \& Interpretation 


\title{
Tank Monitor and Control System (TMACS) Version Description Document (VDD)
}

\section{D.A. Barnes}

CH2M HILL Hanford Group, Inc.

Richland, WA 99352

U.S. Department of Energy Contract DE-AC06-99RL14047

\author{
EDT/ECN: $\quad$ ECN-657305 UC: 605 \\ Cost Center: 74B50 Charge Code: 102608EB00 \\ B\&R Code: EW3120071 . Total Pages: 30
}

Key Words: Version Description Document, VDD, TMACS, Tank Monitor and Con trol

Abstract: This document updates the Version Description Document with the changes incorporated in the Revision 11.2 software installation on the Tank Monitor and Control System (TMACS)

TRADEMARK DISCLAIMER. Reference herein to any specific commercial product, process, or service by trade name, trademark, manufacturer, or otherwise, does not necessarily constitute or imply its endorsement, recommendation, or favoring by the United States Government or any agency thereof or its contractors or subcontractors.

Printed in the United States of America. To obtain copies of this document, contact: Document Control Services, P.O. Box 950, Mailstop H6-08, Richland WA 99352, Phone (509) 372-2420; Fax (509) 376-4989.
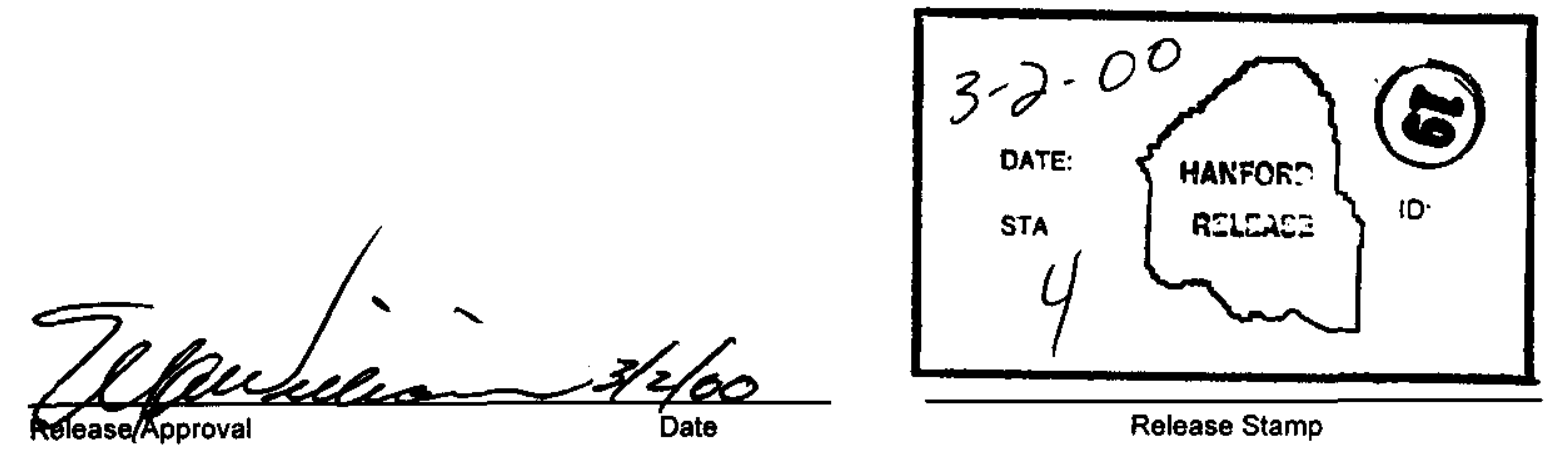

Release Stamp

\section{Approved For Public Release}




\section{RECORD OF REVISION}

(2) Title

Tank Monitor and Control System (TMACS) Version Description Document for Revision 11.1.

CHANGE CONTROL RECORD

\begin{tabular}{|c|c|c|c|}
\hline \multirow{2}{*}{ Revision } & \multirow{2}{*}{ (4) Deseription of Change - Replace, Add, and Delete Pages } & \multicolumn{2}{|c|}{ Authorized for Release } \\
\hline & & (5) Cog. Engr. & (6) Cog. Mgr. \\
\hline 0 & (7) HNF-3538 (EDT 625999) & M. J. Holm & N. W. Kirch \\
\hline 1 & Complete revision per ECN 653556 . & M. J. Holm & N. W. Kirch \\
\hline Ris 2 & Complete revision per ECN 655997. & M. J. Holm & J. G. Field \\
\hline & Wue-ECN-653554 \& ECN-654686 & & 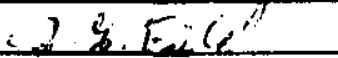 \\
\hline & & & $1 \quad 12.7 \cdot 77$ \\
\hline 8 & Insorporate per F(N- 657305 & P.A. Bosnes & $S, 6, F i 0\rangle$ \\
\hline & & & $3-1-00$ \\
\hline & & for & grisiled \\
\hline & & 3.200 & \\
\hline & & & \\
\hline & & & \\
\hline & & & \\
\hline & & & \\
\hline & & & \\
\hline & & & \\
\hline & & & \\
\hline & & & \\
\hline & & & \\
\hline & & & \\
\hline & & & \\
\hline & & & \\
\hline & & & \\
\hline & & & \\
\hline & & & \\
\hline & & & \\
\hline & & & \\
\hline & & & \\
\hline & & & \\
\hline & & & \\
\hline & & & \\
\hline & & & \\
\hline - & 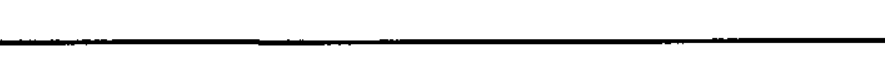 & & $\mathrm{C}_{\mathrm{C}}$ \\
\hline
\end{tabular}


HNF-3538 Rev. 3

Tank Monitor and Control System

(TMACS)

Version Description Document

\author{
Prepared By \\ Ronald R. Wandling \\ Lockheed Martin Services, Inc. \\ For \\ CH2M HILL Hanford Group \\ For \\ US Department of Energy \\ Office of River Protection \\ Richland, Washington
}




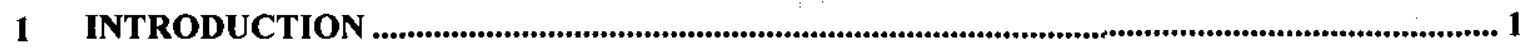

1.1 PURPOSE.

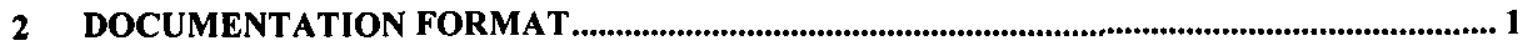

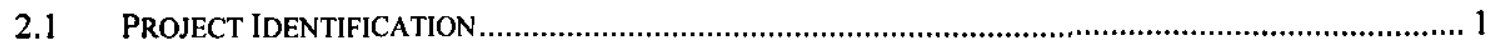

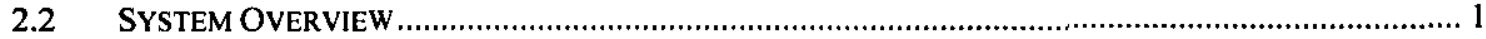

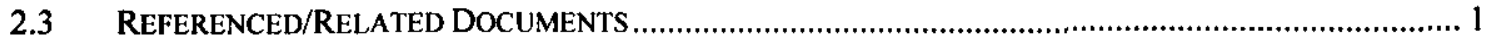

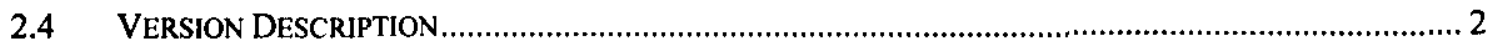

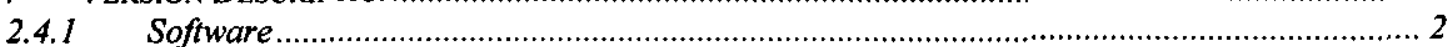

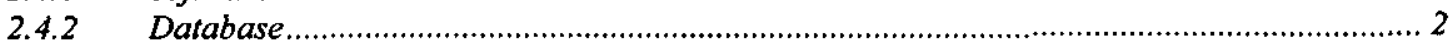

2.4.3 Client Operating System and Environment ..................................................................... 2

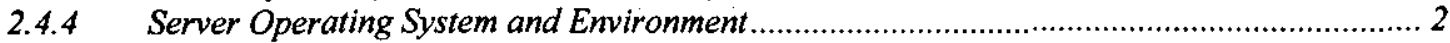

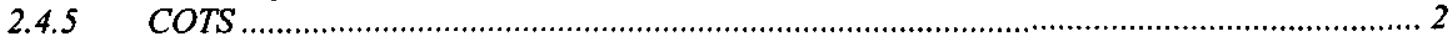

2.4.6 Metadata

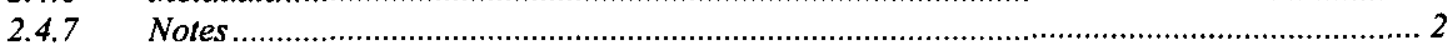

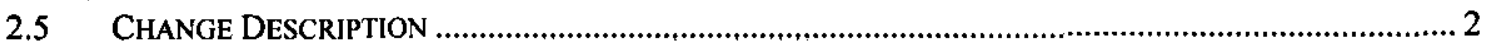

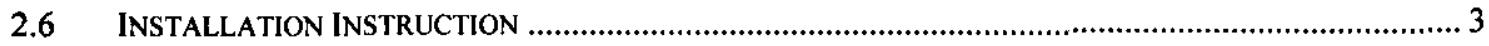

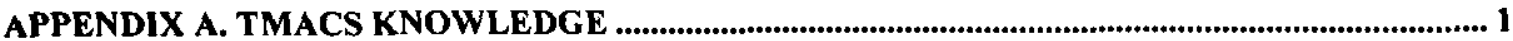

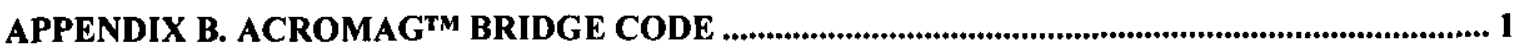

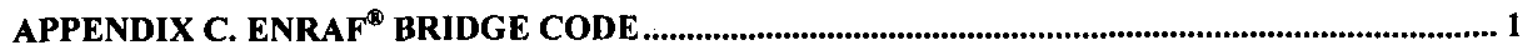

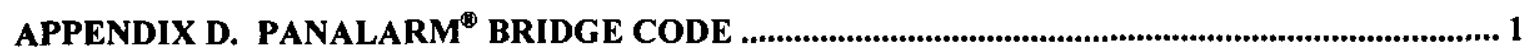

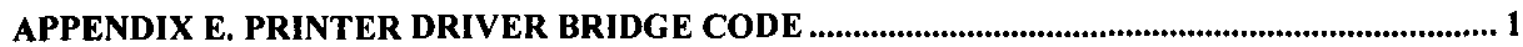

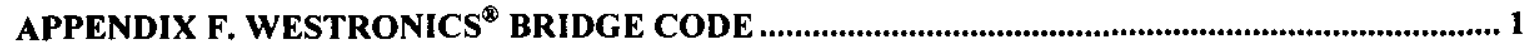

APPENDIX G. WINDOWS NT COMMON CODE....................................................................................

APPENDIX H. BRIDGE CODE EXECUTABLE FILES...................................................................... 1

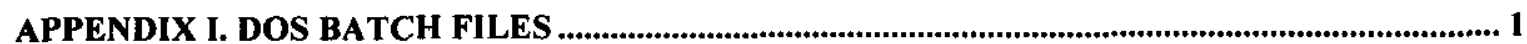




\section{INTRODUCTION}

\subsection{Purpose}

This document contains configuration information for the Tank Monitor and Control System (TMACS) release 11.2. This version was installed in production on February 2, 2000 .

\section{DOCUMENTATION Format}

\subsection{Project Identification}

The Tank Monitor and Control System uses a 3-layer revision descriptor. The descriptor consists of a major revision number, a minor revision number, and a "fix" number, e.g., major revision 5, minor revision 3, and fix 2 would have the identifier 5.3.2.

\subsection{SYSTEM OVERVIEW}

TMACS was established in 1991 under the Tank Farm Instrument Upgrade project to monitor temperatures and surface levels in the Hanford 200 area "watch list" tanks.

\subsection{REFERENCED/RELATED DOCUMENTS}

\begin{tabular}{|l|l|}
\hline System Functional Requirements & WHC-SD-WM-SFR-006, Rev 1 \\
\hline $\begin{array}{l}\text { TMACS As-Built Design } \\
\text { Document }\end{array}$ & WHC-SD-WM-SDD-079, Rev 0 \\
\hline $\begin{array}{l}\text { System Configuration Management } \\
\text { Plan }\end{array}$ & HNF-SD-CSCM-019, Rev 1 \\
\hline $\begin{array}{l}\text { Functional Requirements for the } \\
\text { Acromag I/O Processor Interface } \\
\text { to the Tank Monitor and Control } \\
\text { System (TMACS) }\end{array}$ & HNF-2708, Rev. 0 \\
\hline $\begin{array}{l}\text { Functional Requirements for the } \\
\text { Enraf }{ }^{2} \text { Gauge Interface to the Tank } \\
\text { Monitor and Control System } \\
\text { (TMACS) }\end{array}$ & HNF-2791, Rev. 0 \\
\hline $\begin{array}{l}\text { Functional Requirements for the } \\
\text { Panalarm }{ }^{3} \text { Annunciator System } \\
\text { Interface to the Tank Monitor and } \\
\text { Control System (TMACS) }\end{array}$ & HNF-3053, Rev. 0 \\
\hline $\begin{array}{l}\text { Functional Requirement for the } \\
\text { Westronics }{ }^{4} \text { Interface to the Tank } \\
\text { Monitor and Control System } \\
\text { (TMACS) }\end{array}$ & HNF-2607, Rev. 0 \\
\hline
\end{tabular}

'Acromag is a trademark of Acromag Incorporated, Wixom, Michigan.

${ }^{2}$ Enraf is a registered trademark of Enraf, B.V.

${ }^{3}$ Panalarm is a registered trademark of Ametek, Inc.

${ }^{4}$ Westronics is a registered trademark of Westronics, Inc. 


\subsection{VERSION DESCRIPTION}

\subsubsection{Software}

The main processing performed by TMACS is built using the $\mathrm{G}^{5}$ software product from Gensym. The knowledge bases used are in Appendix A.

Interfaces to field equipment use services written in Microsoft ${ }^{6}$ Visual $^{7}$. The serial port hardware and software were purchased from Digi ${ }^{8}$ International Incorporated. The source code files are listed in Appendix B through Appendix F. The executable files are listed in Appendix $\mathrm{H}$ and the batch files are listed in Appendix I.

\subsubsection{Database}

None.

\subsubsection{Client Operating System and Environment}

The client workstations run WindowsNT ${ }^{9}$ workstation 4.0.

\subsubsection{Server Operating System and Environment}

The WindowsNT ${ }^{\mathrm{TM}}$ server containing the $\mathrm{G2}^{\circledR}$ : software product from Gensym and the interfaces to field equipment runs WindowsNT ${ }^{\mathrm{TM}}$ Server 4.0 with service pack 3 installed.

\subsubsection{COTS}

Telewindows ${ }^{10}$ Revision 4.0 Release 2 is used for remote display of TMACS. It runs on the client workstations.

$\mathrm{G} 2{ }^{\circledR}$ Revision 4.1 Release 1 is used to run the main TMACS processing.

\subsubsection{Metadata}

None.

\subsubsection{Notes}

Controlled source files are kept in SourceSafe on the IIUDO computer.

\subsection{Change Description}

The following are the service request incorporated in TMACS revision 11.2.

\begin{tabular}{|l|l|}
\hline SR \# & Abbreviated Description \\
\hline & \\
\hline 103 & Make the GSI Data Service Priority lower than the Processing Rule Priority \\
\hline
\end{tabular}

${ }^{5} \mathrm{G} 2$ is a registered trademark of the Gensym Corporation.

${ }^{6} \mathrm{Microsoft}$ is a registered trademark of the Microsoft Corporation.

${ }^{7} \mathrm{~V}$ isual $\mathrm{C}$ is a trademark of the Microsoft Corporation.

${ }^{8}$ Digi is a trademark of Digi International Incorporated.

${ }^{9}$ WindowsNT is a trademark of Microsoft Corporation.

${ }^{10}$ Telewindows is a registered trademark of the Gensym Corporation. 
HNF-3538 Rev. 3

\begin{tabular}{|l|l|}
\hline \multicolumn{1}{|c|}{ SR \# } & \multicolumn{1}{|c|}{ Abbreviated Description } \\
\hline 132 & Add individual trend graphs for discrete sensors \\
\hline 166 & Modify TMACS to display the color red for a K-Basin Communications Alarm \\
\hline 403 & Modify TMACS to display the sensor limits for a sensor. \\
\hline 434 & Modify TMACS To Read Pressure Sensors through ENRAF CIU device \\
\hline 752 & Modify The ENARF Driver To Properly Handle The “!” Commands \\
\hline 856 & $\begin{array}{l}\text { Devise A Method To Notify The Operator When An ENRAF Level Gauge Has A } \\
\text { Status Other Than “-“ }\end{array}$ \\
\hline 857 & Correct Unknown Sensor Report To Display Only Sensors Associated With Tanks. \\
\hline 858 & $\begin{array}{l}\text { Modify The Suspect IO Station Report To Not Display Station With A Polling } \\
\text { Frequency Of Zero. }\end{array}$ \\
\hline 860 & $\begin{array}{l}\text { Correct Problem Of Old Print Screen Button Being Displayed For Multiple Sensor } \\
\text { Trends }\end{array}$ \\
\hline 862 & $\begin{array}{l}\text { Correct Problem Where Sensor With Alarm Processing Turn Off Do Not Restore To } \\
\text { Correct Color When Communications Are Re-Established. }\end{array}$ \\
\hline 865 & Set Polling Frequency Of Double Shell Tanks To 10 Minutes Or Less. \\
\hline 867 & Add Ability To Place A Sensor Into Test Mode. \\
\hline 869 & Configure TMACS To Have ENRAF Level Gauges For Tanks BY102 And S110 \\
\hline 871 & Change Gauge Addresses for ENRAF Level Devices in Tanks AP105-AP108 \\
\hline 873 & Create Report That Displays Active Sensors With Alarm Processing Disabled \\
\hline
\end{tabular}

\subsection{INSTALLATION INSTRUCTION}

TMACS bridges are installed as services on the TMACSPROD server.

The $\mathrm{G} 2{ }^{\otimes}$ executable is installed as a program on the TMACSPROD server.

TMACS G $2{ }^{\circledast}$ code is kept in a series of knowledge bases that are transferred to TMACSPROD for operation.

Telewindows ${ }^{\circledR}$ software is installed on the client workstations and requires a hardware key. A new version of Telewindows ${ }^{\circledR}$ is installed when a new, vendor-supplied release of $\mathrm{G} 2{ }^{\mathbb{B}}$ is installed. 


\section{Appendix A. TMACS Knowledge}

These files are stored in the \$/TMACS/Release Files/11.1/KnowledgeBases directory.

$* * * * * * * * * * * * * * * * *$ enraf-root.kb $* * * * * * * * * * * * * * * * *$

Label: 11.2

User: Steve Date: 2/01/:0 Time: 4:11p

Labeled '11.2'

Label comment:

$* * * * * * * * * * * * * * * * *$ htff.kb

Label: 11.2

User: Steve

Date: $2 / 01 /: 0$ Time: $4: 11 \mathrm{p}$

Labeled '11.2'

Label comment:

$* * * * * * * * * * * * * * * * *$ acromag-root. $\mathrm{kb}$

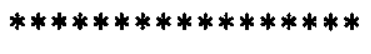

Label: 11.2

User: Steve

Date: $2 / 01 /: 0$ Time: $3: 34 p$

Labeled '11.2'

Label comment:

$* * * * * * * * * * * * * * * * *$ data-recovery. $\mathrm{kb}$

Label: 11.2

User: Steve

Date: $2 / 01 /: 0$ Time: $3: 34 p$

Labeled '11.2'

Label comment:

$* * * * * * * * * * * * * * * * * \mathrm{~g} 2$-database. $\mathrm{kb} * * * * * * * * * * * * * * * * *$

Label: 11.2

User: Steve Date: $2 / 01 /: 0$ Time: $3: 34 \mathrm{p}$

Labeled '11.2'

Label comment:

$* * * * * * * * * * * * * * * * *$ gensym-raised-buttons.kb

$* * * * * * * * * * * * * * * * *$

Label: 11.2

User: Steve

Date: $2 / 01 /: 0$ Time: $3: 34 \mathrm{p}$

Labeled '11.2'

Label comment: 
$* * * * * * * * * * * * * * * * *$ gfi-mod.kb

Label: 11.2

User: Steve Date: 2/01/:0 Time: $3: 34 \mathrm{p}$

Labeled '11.2'

Label comment:

$* * * * * * * * * * * * * * * * *$ level. $\mathrm{kb} * * * * * * * * * * * * * * * * *$

Label: 11.2

User: Steve Date: $2 / 01 /: 0$ Time: $3: 34 \mathrm{p}$

Labeled '11.2'

Label comment:

$* * * * * * * * * * * * * * * * *$ odbc-root. $\mathrm{kb} * * * * * * * * * * * * * * * * *$

Label: 11.2

User: Steve Date: 2/01/:0 Time: $3: 34 \mathrm{p}$

Labeled '11.2'

Label comment:

$* * * * * * * * * * * * * * * * *$ panalarm-root.kb $* * * * * * * * * * * * * * * * *$

Label: 11.2

User: Steve Date: 2/01/:0 Time: $3: 34 \mathrm{p}$

Labeled '11.2'

Label comment:

$* * * * * * * * * * * * * * * * *$ printer-root.kb $* * * * * * * * * * * * * * * * *$

Label: 11.2

User: Steve Date: $2 / 01 /: 0$ Time: $3: 34 \mathrm{p}$

Labeled '11.2'

Label comment:

$* * * * * * * * * * * * * * * * *$ surveillance-limit-root. $\mathrm{kb}$

Label: 11.2

User: Steve Date: 2/01/:0 Time: $3: 34 \mathrm{p}$

Labeled '11.2'

Label comment:

Label: 11.2

User: Steve

Labeled '11.2'

$$
\text { sys-mod.kb }
$$

Date: $2 / 01 /: 0$ Time: $3: 34 \mathrm{p}$ 
Label comment:

$* * * * * * * * * * * * * * * * *$ tmacs-definitions.kb

Label: 11.2

User: Steve

Date: $2 / 01 /: 0$ Time: $3: 34 \mathrm{p}$

Labeled '11.2'

Label comment:

$* * * * * * * * * * * * * * * * *$ TMACS_Main.kb

Label: 11.2

User: Steve Date: 2/01/:0 Time: $3: 34 \mathrm{p}$

Labeled '11.2'

Label comment:

$* * * * * * * * * * * * * * * * *$ uilroot. $\mathrm{kb}$

Label: 11.2

User: Steve

Date: $2 / 01 /: 0$ Time: $3: 34 p$

Labeled '11.2'

Label comment:

$* * * * * * * * * * * * * * * * *$ westronics-root.kb

Label: 11.2

User: Steve

Date: $2 / 01 /: 0$ Time: $3: 34 p$

Labeled '11.2'

Label comment:

$* * * * * * * * * * * * * * * * *$ point-process.kb $* * * * * * * * * * * * * * * * *$

Label: 11.2

User: Steve Date: $2 / 01 /: 0$ Time: $3: 34 p$

Labeled '11.2'

Label comment:

$* * * * * * * * * * * * * * * * *$ tmacs-lib.kb

Label: 11.2

User: Steve

Date: $2 / 01 /: 0$ Time: $3: 34 \mathrm{p}$

Labeled '11.2'

Label comment: 


\section{Appendix B. Acromagrm Bridge Code}

These files are stored in the \$/TMACS/Bridges/Acromag ${ }^{\text {TM }}$ directory.

\$/TMACS/Bridges/Acromag

$* * * * * * * * * * * * * * * * *$ acro_exts.h $* * * * * * * * * * * * * * * * *$

Label: 11.2

User: Steve

Date: $2 / 01 /: 0$ Time: $12: 11 \mathrm{p}$

Labeled '11.2'

Label comment:

$* * * * * * * * * * * * * * * * *$ acro_main.c $* * * * * * * * * * * * * * * * *$

Label: 11.2

User: Steve

Date: $2 / 01 /: 0$ Time: $12: 11 \mathrm{p}$

Labeled '11.2'

Label comment:

$* * * * * * * * * * * * * * * * *$ acro_vars.h

Label: 11.2

User: Steve

Date: $2 / 01 /: 0$ Time: $12: 11 \mathrm{p}$

Labeled '11.2'

Label comment:

$* * * * * * * * * * * * * * * * *$ gsi_abpi_codes.h

Label: 11.2

User: Steve

Date: $2 / 01 /: 0$ Time: $12: 11 \mathrm{p}$

Labeled '11.2'

Label comment:

$* * * * * * * * * * * * * * * * *$ md_linkio.h

Label: 11.2

User: Steve

Date: $2 / 01 /: 0$ Time: $12: 11 \mathrm{p}$

Labeled '11.2'

Label comment:

$* * * * * * * * * * * * * * * * *$ resource.h

Label: 11.2

User: Steve

Date: $2 / 01 /: 0$ Time: $12: 11 p$

Labeled '11.2'

Label comment: 
$* * * * * * * * * * * * * * * * *$ SERVICE.H

Label: 11.2

User: Steve

Date: $2 / 01 /: 0$ Time: $12: 11 \mathrm{p}$

Labeled '11.2'

Label comment:

$* * * * * * * * * * * * * * * * *$ acro.h

Label: 11.2

User: Steve

Date: 2/01/:0 Time: 12:11p

Labeled '11.2'

Label comment:

$* * * * * * * * * * * * * * * * *$ acro_bridge.c $* * * * * * * * * * * * * * * * *$

Label: 11.2

User: Steve

Date: 2/01/:0 Time: 12:11p

Labeled '11.2'

Label comment:

$* * * * * * * * * * * * * * * * *$ acro_link.c $* * * * * * * * * * * * * * * * *$

Label: 11.2

User: Steve

Labeled '11.2'

Date: 2/01/:0 Time: 12:11p

Label comment:

Label: 11.2

User: Steve Date: 2/01/:0 Time: 12:11p

Labeled '11.2'

Label comment:

$* * * * * * * * * * * * * * * * *$ acro_main.dsw $* * * * * * * * * * * * * * * * *$

Label: 11.2

User: Steve

Labeled '11.2'

Date: 2/01/:0 Time: 12:11p

Label comment:

$* * * * * * * * * * * * * * * * *$ acro_main.ncb

Label: 11.2 
User: Steve Date: 2/01/:0 Time: $12: 11 \mathrm{p}$

Labeled '11.2'

Label comment:

$* * * * * * * * * * * * * * * * *$ acro_main.plg

Label: 11.2

User: Steve Date: 2/01/:0 Time: 12:11p

Labeled '11.2'

Label comment:

$* * * * * * * * * * * * * * * * *$ acro_rpcs.c $* * * * * * * * * * * * * * * * *$

Label: 11.2

User: Steve Date: $2 / 01 /: 0$ Time: $12: 11 \mathrm{p}$

Labeled '11.2'

Label comment:

$* * * * * * * * * * * * * * * * *$ acromessage.aps

Label: 11.2

User: Steve

Date: $2 / 01 /: 0$ Time: $12: 11 \mathrm{p}$

Labeled '11.2'

Label comment:

$* * * * * * * * * * * * * * * * *$ acromessage.rc

Label: 11.2

User: Steve Date: $2 / 01 /: 0$ Time: $12: 11 \mathrm{p}$

Labeled '11.2'

Label comment:

$* * * * * * * * * * * * * * * * *$ gsi_utils.c $* * * * * * * * * * * * * * * * *$

Label: 11.2

User: Steve Date: $2 / 01 /: 0$ Time: $12: 11 \mathrm{p}$

Labeled '11.2'

Label comment:

$* * * * * * * * * * * * * * * * *$ md_errors.h

Label: 11.2

User: Steve

Date: $2 / 01 /: 0$ Time: $12: 11 \mathrm{p}$

Labeled '11.2'

Label comment: 
$* * * * * * * * * * * * * * * * * \mathrm{mf}$ functions.c

Label: 11.2

User: Steve Date: 2/01/:0 Time: 12:11p

Labeled '11.2'

Label comment:

$* * * * * * * * * * * * * * * * *$ set check init.c $* * * * * * * * * * * * * * * *$

Label: 11.2

User: Steve Date: 2/01/:0 Time: 12:11p

Labeled '11.2'

Label comment:

Label: 11.2

User: Steve

Labeled '11.2'

acro_main.opt

Label comment: 


\section{Appendix C. Enraf ${ }^{\text {Bridge Code }}$}

These files are stored in the \$/TMACS/Bridges/Enraf ${ }^{\circledR}$ directory.

\$/TMACS/Bridges/Enraf

****************** check_enraf_init.c $* * * * * * * * * * * * * * * * *$

Label: 11.2

User: Steve Date: 2/01/:0 Time: 12:09p

Labeled '11.2'

Label comment:

Add Rosmount pressure to driver.

$* * * * * * * * * * * * * * * * *$ enraf_link.c $* * * * * * * * * * * * * * * * *$

Label: 11.2

User: Steve

Labeled '11.2'

Label comment:

Add Rosmount pressure to driver.

$* * * * * * * * * * * * * * * * *$ enraf main.c $* * * * * * * * * * * * * * * * *$

Label: 11.2

User: Steve

Date: $2 / 01 /: 0$ Time: 12:09p

Labeled '11.2'

Label comment:

Add Rosmount pressure to driver.

$* * * * * * * * * * * * * * * * *$ enraf_main.dsp $* * * * * * * * * * * * * * * * *$

Label: 11.2

User: Steve Date: $2 / 01 /: 0$ Time: $12: 09 p$

Labeled '11.2'

Label comment:

Add Rosmount pressure to driver.

$* * * * * * * * * * * * * * * * *$ enraf_main.dsw

Label: 11.2

User: Steve Date: 2/01/:0 Time: 12:09p

Labeled '11.2'

Label comment:

Add Rosmount pressure to driver.

$* * * * * * * * * * * * * * * * *$ enraf_utils.c

Label: 11.2

User: Steve

Labeled '11.2'

Label comment:

Add Rosmount pressure to driver.

***************** md_errors.h

Label: 11.2

User: Steve

Labeled ' 11.2 '

Label comment:

Add Rosmount pressure to driver. 


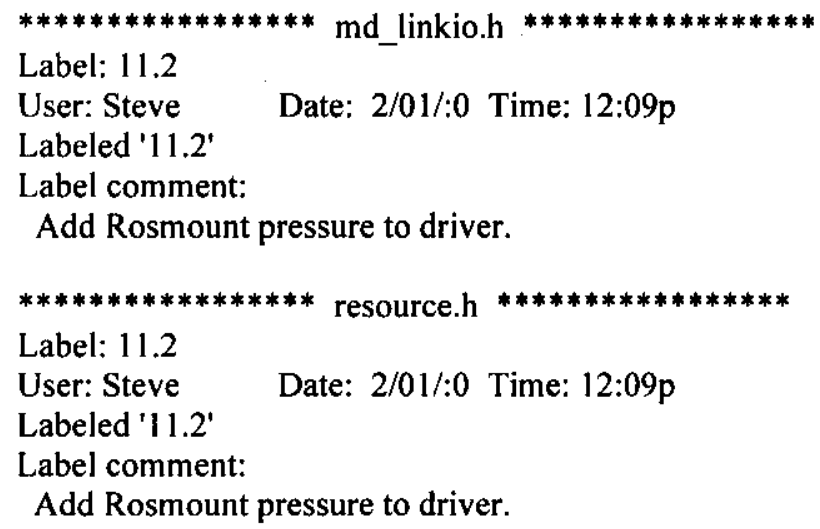

Label: 11.2

User: Steve Date: 2/01/:0 Time: 12:09p

Labeled '11.2'

Label comment:

Add Rosmount pressure to driver.

$* * * * * * * * * * * * * * * * *$ enraf.aps $* * * * * * * * * * * * * * * * *$

Label: 11.2

User: Steve Date: 2/01/:0 Time: 12:09p

Labeled '11.2'

Label comment:

Add Rosmount pressure to driver.

$* * * * * * * * * * * * * * * * *$ enraf.h $* * * * * * * * * * * * * * * * *$

Label: 11.2

User: Steve

Date: $2 / 01 /: 0$ Time: 12:09p

Labeled '11.2'

Label comment:

Add Rosmount pressure to driver.

$* * * * * * * * * * * * * * * * *$ enraf.rc $* * * * * * * * * * * * * * * * *$

Label: 11.2

User: Steve

Labeled '1 1.2'

Date: $2 / 01 /: 0$ Time: $12: 09 p$

Label comment:

Add Rosmount pressure to driver.

$* * * * * * * * * * * * * * * * *$ enraf_bridge.c $* * * * * * * * * * * * * * * * *$

Label: 11.2

User: Steve Date: 2/01/:0 Time: 12:09p

Labeled '11.2'

Label comment:

Add Rosmount pressure to driver.

$* * * * * * * * * * * * * * * * *$ enraf_functions.c $* * * * * * * * * * * * * * * * *$

Label: 11.2

User: Steve

Date: $2 / 01 /: 0$ Time: 12:09p

Labeled '11.2'

Label comment:

Add Rosmount pressure to driver. 
$* * * * * * * * * * * * * * * * *$ enraf main.ncb

Label: 11.2

User: Steve

Date: 2/01/:0 Time: 12:09p

Labeled '11.2'

Label comment:

Add Rosmount pressure to driver.

Label: 11.2

User: Steve

Labeled '11.2'

Label comment:

Add Rosmount pressure to driver.

$* * * * * * * * * * * * * * * * *$ enraf_main.plg $* * * * * * * * * * * * * * * * *$

Label: 11.2

User: Steve

Date: $2 / 01 /: 0$ Time: $12: 09 p$

Labeled '11.2'

Label comment:

Add Rosmount pressure to driver.

$* * * * * * * * * * * * * * * * *$ enraf_rpcs.c $* * * * * * * * * * * * * * * * *$

Label: 11.2

User: Steve

Labeled '11.2'

Label comment:

Add Rosmount pressure to driver. 


\section{Appendix D. Panalarm ${ }^{\circledR}$ Bridge Code}

These files are stored in the $\$ /$ TMACS/Bridges/Panalarm $®$ directory.

\$/TMACS/Bridges/Panalarm

$* * * * * * * * * * * * * * * * *$ gsi_main.c $* * * * * * * * * * * * * * * * *$

Label: 11.2

User: Steve Date: $2 / 01 /: 0$ Time: $12: 14 \mathrm{p}$

Labeled '11.2'

Label comment:

$* * * * * * * * * * * * * * * * *$ gsi panalm_codes.h

Label: 11.2

User: Steve

Date: $2 / 01 /: 0$ Time: $12: 14 \mathrm{p}$

Labeled '11.2'

Label comment:

$* * * * * * * * * * * * * * * * *$ gsi_utils.c $* * * * * * * * * * * * * * * * *$

Label: 11.2

User: Steve Date: $2 / 01 /: 0$ Time: $12: 14 \mathrm{p}$

Labeled '11.2'

Label comment:

$* * * * * * * * * * * * * * * * *$ gsi_utils.h $\mathrm{h} * * * * * * * * * * * * * * * *$

Label: 11.2

User: Steve

Labeled '11.2'

Date: $2 / 01 /: 0$ Time: $12: 14 \mathrm{p}$

Label comment:

$* * * * * * * * * * * * * * * * * \mathrm{md}$ errors.h

Label: 11.2

User: Steve Date: $2 / 01 /: 0$ Time: $12: 14 p$

Labeled '11.2'

Label comment:

$* * * * * * * * * * * * * * * * *$ panalm. $h$

Label: 11.2

User: Steve

Labeled '11.2'

Date: $2 / 01 /: 0$ Time: $12: 14 \mathrm{p}$

Label comment: 
Label: 11.2

User: Steve

panalm_link.c

$* * * * * * * * * * * * * * * * *$

Labeled '11.2'

Label comment:

$* * * * * * * * * * * * * * * * *$ panalm_utils.c

Label: 11.2

User: Steve

Labeled '11.2'

Date: 2/01/:0 Time: 12:14p

Label comment:

$* * * * * * * * * * * * * * * * *$ resource.h $* * * * * * * * * * * * * * * * *$

Label: 11.2

User: Steve Date: 2/01/:0 Time: 12:14p

Labeled '11.2'

Label comment:

$* * * * * * * * * * * * * * * * *$ set_check_init.c $* * * * * * * * * * * * * * * * *$

Label: 11.2

User: Steve

Labeled '11.2'

Date: $2 / 01 /: 0$ Time: $12: 14 \mathrm{p}$

Label comment:

Label: 11.2

User: Steve

Labeled '11.2'

Date: $2 / 01 /: 0$ Time: $12: 14 p$

Label comment:

$* * * * * * * * * * * * * * * * *$ gsi main.dsw

Label: 11.2

User: Steve

Labeled '11.2'

Label comment:

Date: 2/01/:0 Time: 12:14p

Label: 11.2 
User: Steve

Labeled '11.2'

Date: $2 / 01 /: 0$ Time: $12: 14 p$

Label comment:

Label: 11.2

User: Steve

gsi_main.opt

Labeled '11.2'

Label comment:

Label: 11.2

User: Steve

Labeled '11.2'

Label comment:

Date: 2/01/:0 Time: 12:14p

Label: 11.2

User: Steve

Labeled '11.2'

gsi_main.plg

Label comment:

$$
\text { panalarm.aps }
$$

Date: $2 / 01 /: 0$ Time: $12: 14 p$

Label: 11.2

User: Steve

Date: $2 / 01 /: 0$ Time: $12: 14 p$

Labeled '11.2'

Label comment:

Label: 11.2

User: Steve

Labeled '11.2'

Label comment:

$$
\text { panalarm.rc } * * * * * * * * * * * * * * * * *
$$

Date: 2/01/:0 Time: 12:14p

Label: 11.2

User: Steve

Labeled '11.2'

panalm_bridge.c

Date: 2/01/:0 Time: 12:14p

Label comment: 
HNF-3538 Rev. 3

$* * * * * * * * * * * * * * * * *$ SERVICE.H $* * * * * * * * * * * * * * * * *$

Label: 11.2

User: Steve Date: 2/01/:0 Time: 12:14p

Labeled '11.2'

Label comment:

D -4 


\section{Appendix E. Printer Driver Bridge Code}

These files are stored in the \$/TMACS/Bridges/Printer directory.

\$/TMACS/Bridges/Printer

***************** alarmprinter.aps

Label: 11.2

User: Steve

Date: 2/01/:0 Time: 12:14p

Labeled '11.2'

Label comment:

$* * * * * * * * * * * * * * * *$ gsi_utils.c

Label: 11.2

User: Steve Date: $2 / 01 /: 0$ Time: 12:14p

Labeled '11.2'

Label comment:

$* * * * * * * * * * * * * * * * *$ gsi_utils.h

Label: 11.2

User: Steve

Labeled '11.2'

Label comment:

Date: $2 / 01 /: 0$ Time: $12: 14 p$

$* * * * * * * * * * * * * * * * *$ printer.h

Label: 11.2

User: Steve

Date: $2 / 01 /: 0$ Time: 12:14p

Labeled '11.2'

Label comment:

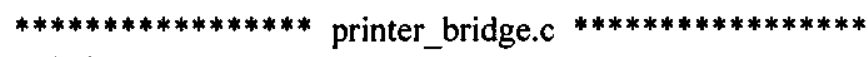

Label: 11.2

User: Steve

Labeled '11.2'

Label comment:

Date: $2 / 01 /: 0$ Time: $12: 14 p$

***************** printer_main.c

Label: 11.2

User: Steve

Labeled '11.2'

Date: $2 / 01 /: 0$ Time: $12: 14 p$

Label comment:

***************** printer_main.dsp $* * * * * * * * * * * * * * * * *$

Label: 11.2

User: Steve

Labeled '11.2'

Date: $2 / 01 /: 0$ Time: $12: 14 p$ 
Label comment:

$* * * * * * * * * * * * * * * * *$ printer_main.dsw $* * * * * * * * * * * * * * * * *$

Label: 11.2

User: Steve

Date: $2 / 01 /: 0$ Time: 12:14p

Labeled '11.2'

Label comment:

$* * * * * * * * * * * * * * * * *$ resource. $\mathrm{h} * * * * * * * * * * * * * * * * *$

Label: 11.2

User: Steve

Date: $2 / 01 /: 0$ Time: $12: 14 \mathrm{p}$

Labeled '11.2'

Label comment:

$* * * * * * * * * * * * * * * * *$ SERVICE.H $* * * * * * * * * * * * * * * * *$

Label: 11.2

User: Steve

Labeled '11.2'

Date: $2 / 01 /: 0$ Time: $12: 14 p$

Label comment:

$* * * * * * * * * * * * * * * * *$ alarmprinter.rc $* * * * * * * * * * * * * * * * *$

Label: 11.2

User: Steve Date: $2 / 01 /: 0$ Time: $12: 14 p$

Labeled '11.2'

Label comment:

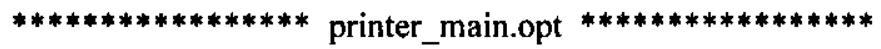

Label: 11.2

User: Steve Date: $2 / 01 /: 0$ Time: $12: 14 \mathrm{p}$

Labeled '11.2'

Label comment:

***************** printer_main.plg

Label: 11.2

User: Steve

Labeled '1 1.2'

Label comment:

Date: $2 / 01 /: 0$ Time: 12:14p 


\section{Appendix F. Westronics ${ }^{\circledR}$ Bridge Code}

These files are stored in the \$/TMACS/Bridges/Westronics $\otimes$ directory,

\$/TMACS/Bridges/Westronics

$* * * * * * * * * * * * * * * * *$ gsi_abpi_codes.h $* * * * * * * * * * * * * * * * *$

Label: 11.2

User: Steve

Date: $2 / 01 /: 0$ Time: $12: 15 \mathrm{p}$

Labeled '11.2'

Label comment:

$* * * * * * * * * * * * * * * * *$ gsi_utils.c

Label: 11.2

User: Steve

Date: $2 / 01 /: 0$ Time: $12: 15 \mathrm{p}$

Labeled '11.2'

Label comment:

$* * * * * * * * * * * * * * * * *$ md_errors.h

Label: 11.2

User: Steve

Date: $2 / 01 /: 0$ Time: $12: 15 p$

Labeled '11.2'

Label comment:

Label: 11.2

User: Steve

Date: $2 / 01 /: 0$ Time: $12: 15 p$

Labeled '11.2'

Label comment:

$* * * * * * * * * * * * * * * * *$ resource.h

$* * * * * * * * * * * * * * * * *$

Label: 11.2

User: Steve

Date: $2 / 01 /: 0$ Time: $12: 15 p$

Labeled '11.2'

Label comment:

$* * * * * * * * * * * * * * * * *$ SERVICE.H

Label: 11.2

User: Steve

Date: $2 / 01 /: 0$ Time: $12: 15 p$

Labeled '11.2'

Label comment: 
Label: 11.2

User: Steve

Date: 2/01/:0 Time: 12:15p

Labeled '11.2'

Label comment:

$* * * * * * * * * * * * * * * * *$ west.h $* * * * * * * * * * * * * * * * *$

Label: 11.2

User: Steve

Date: $2 / 01 /: 0$ Time: $12: 15 p$

Labeled '11.2'

Label comment:

Label: 11.2

User: Steve

Labeled '11.2'

Label comment:

$* * * * * * * * * * * * * * * * *$ west link.c

Label: 11.2

User: Steve

Date: $2 / 01 /: 0$ Time: $12: 15 p$

Labeled '11.2'

Label comment:

$* * * * * * * * * * * * * * * * *$ west_main.c ${ }^{* * * * * * * * * * * * * * * * * *}$

Label: 11.2

User: Steve Date: 2/01/:0 Time: 12:15p

Labeled '11.2'

Label comment:

$* * * * * * * * * * * * * * * * *$ west_main.dsp $* * * * * * * * * * * * * * * * *$

Label: 11.2

User: Steve Date: 2/01/:0 Time: 12:15p

Labeled '11.2'

Label comment:

$* * * * * * * * * * * * * * * *$ west_main.dsw

Label: 11.2

User: Steve Date: 2/01/:0 Time: 12:15p 
Labeled '11.2'

Label comment:

$* * * * * * * * * * * * * * * * *$ west_main.plg

Label: 11.2

User: Steve

Date: $2 / 01 /: 0$ Time: $12: 15 p$

Labeled '11.2'

Label comment:

Label: 11.2

User: Steve

Date: $2 / 01 /: 0$ Time: $12: 15 \mathrm{p}$

Labeled '11.2'

Label comment:

$* * * * * * * * * * * * * * * * *$ westronic.rc

Label: 11.2

User: Steve

Date: $2 / 01 /: 0$ Time: $12: 15 p$

Labeled '11.2'

Label comment:

$* * * * * * * * * * * * * * * * *$ west main.ncb

Label: 11.2

User: Steve

Date: $2 / 01 /: 0$ Time: $12: 15 p$

Labeled '11.2'

Label comment:

$* * * * * * * * * * * * * * * * *$ west_main.opt

Label: 11.2

User: Steve Date: $2 / 01 /: 0$ Time: $12: 15 p$

Labeled '11.2'

Label comment:

$* * * * * * * * * * * * * * * * *$ westronic.aps $* * * * * * * * * * * * * * * * *$

Label: 11.2

User: Steve

Date: $2 / 01 /: 0$ Time: $12: 15 p$

Labeled '11.2'

Label comment: 


\section{Appendix G. Windows NT Common Code}

These Files are stored in the \$/TMACS/Bridges/WinNT Common Code directory.

\$/TMACS/Bridges/WinNT Common Code

$* * * * * * * * * * * * * * * * *$ MSG00001.bin *****************

Label: 11.2

User: Steve Date: $2 / 01 /: 0$ Time: $12: 15 \mathrm{p}$

Labeled '11.2'

Label comment:

$* * * * * * * * * * * * * * * * *$ SERVICE.C

Label: 11.2

User: Steve Date: $2 / 01 /: 0$ Time: $12: 15 \mathrm{p}$

Labeled '11.2'

Label comment:

$* * * * * * * * * * * * * * * * *$ servicemain.c $* * * * * * * * * * * * * * * * *$

Label: 11.2

User: Steve

Date: $2 / 01 /: 0$ Time: $12: 15 \mathrm{p}$

Labeled '11.2'

Label comment:

$* * * * * * * * * * * * * * * * *$ servicemsg.aps

Label: 11.2

User: Steve Date: $2 / 01 /: 0$ Time: $12: 15 p$

Labeled '11.2'

Label comment:

$* * * * * * * * * * * * * * * * *$ servicemsg.h $* * * * * * * * * * * * * * * * *$

Label: 11.2

User: Steve Date: 2/01/:0 Time: 12:15p

Labeled '11.2'

Label comment:

$* * * * * * * * * * * * * * * * *$ servicemsg.mc $* * * * * * * * * * * * * * * * *$

Label: 11.2

User: Steve

Date: $2 / 01 /: 0$ Time: $12: 15 p$

Labeled '11.2'

Label comment: 
HNF-3538 Rev. 3

$* * * * * * * * * * * * * * * * *$ servicemsg.rc $* * * * * * * * * * * * * * * * *$

Label: 11.2

User: Steve Date: $2 / 01 /: 0$ Time: $12: 15 p$

Labeled '11.2'

Label comment:

***************** set_physio_nt.c

Label: 11.2

User: Steve

Date: $2 / 01 /: 0$ Time: $12: 15 p$

Labeled '11.2'

Label comment: 


\section{Appendix H. Bridge Code Executable Files}

These files are stored in the $\$ /$ TMACS/Release Files/11.1/Bridge Executables directory.

\$/TMACS/Release Files/11.2/Bridge Executables

$* * * * * * * * * * * * * * * * *$ acromag_driver.exe $* * * * * * * * * * * * * * * * *$

Label: 11.2

User: Steve

Date: 2/01/:0 Time: 4:50p

Labeled '11.2'

Label comment:

$* * * * * * * * * * * * * * * * *$ enraf_driver.exe

Label: 11.2

User: Steve

Date: $2 / 01 /: 0$ Time: $4: 50 p$

Labeled '11.2'

Label comment:

$* * * * * * * * * * * * * * * * *$ panalm_driver.exe $* * * * * * * * * * * * * * * * *$

Label: 11.2

User: Steve Date: 2/01/:0 Time: $4: 50 p$

Labeled '11.2'

Label comment:

$* * * * * * * * * * * * * * * * *$ tmacs printer_driver.exe

Label: 11.2

User: Steve

Date: $2 / 01 /: 0$ Time: $4: 50 \mathrm{p}$

Labeled '11.2'

Label comment:

$* * * * * * * * * * * * * * * * *$ westronic_driver.exe

Label: 11.2

User: Steve

Labeled '11.2'

Date: $2 / 01 /: 0$ Time: $4: 50 p$

Label comment: 
Appendix I. DOS Batch Files

These files are stored in the \$/TMACS/TMACSTools.

\$/TMACS/TMACSTools

$* * * * * * * * * * * * * * * * *$ Version $10 * * * * * * * * * * * * * * * * *$

Label: 11.2

User: Ron

Date: $2 / 03 /: 0$ Time: $2: 43 p$

Labeled '11.2'

Label comment:

$* * * * * * * * * * * * * * * * *$ startsrv.bat

Label: 11.0

User: Ron

Date: 4/23/99 Time: 9:06a

Labeled '11.0'

Label comment:

Batch Files Used in NT version of TMACS

$* * * * * * * * * * * * * * * * *$ stopsrv.bat

Label: 11.0

User: Ron

Date: 4/23/99 Time: 9:06a

Labeled '11.0'

Label comment:

Batch Files Used in NT version of TMACS

$* * * * * * * * * * * * * * * * *$ TMACSPrint.bat

Label: 11.0

User: Ron

Date: 4/23/99 Time: 9:06a

Labeled '11.0'

Label comment:

Batch Files Used in NT version of TMACS 